\title{
A Low-Noise, Wideband Preamplifier for a Fourier-Transform Ion Cyclotron Resonance Mass Spectrometer
}

\author{
Raman Mathur, ${ }^{a, b}$ Ronald W. Knepper, ${ }^{a}$ and Peter B. O'Connor ${ }^{\mathrm{a}, \mathrm{b}, \mathrm{c}}$ \\ a Department of Electrical and Computer Engineering, Boston University, Boston, Massachusetts, USA \\ ${ }^{\mathrm{b}}$ Cardiovascular Proteomics Center, Boston University, Boston, Massachusetts, USA \\ ${ }^{\text {c }}$ Mass Spectrometry Resource, Department of Biochemistry, Boston University, Boston, Massachusetts, USA
}

FTMS performance parameters such as limits of detection, dynamic range, sensitivity, and even mass accuracy and resolution can be greatly improved by enhancing its detection circuit. An extended investigation of significant design considerations for optimal signal-to-noise ratio in an FTMS detection circuit are presented. A low noise amplifier for an FTMS is developed based on the discussed design rules. The amplifier has a gain of $\approx 3500$ and a bandwidth of 10 $\mathrm{kHz}$ to $1 \mathrm{MHz}$ corresponding to $\mathrm{m} / z$ range of $100 \mathrm{Da}$ to $10 \mathrm{kDa}$ (at 7 Tesla). The performance of the amplifier was tested on a MALDI-FTMS, and has demonstrated a 25-fold reduction in noise in a mass spectrum of $\mathrm{C}_{60}$ compared with that of a commercial amplifier. (J Am Soc Mass Spectrom 2007, 18, 2233-2241) (C 2007 American Society for Mass Spectrometry

$\mathrm{M}$ odern mass spectrometry plays a critical role in the analysis of biological proteins and other compounds of significance. In addition to structural information, a mass spectrometer can also be used to study interactions between proteins [1-4]. However, these studies pose considerable challenges due to the complexity of the cellular proteome. Some of the most effective mass spectrometers are time-of-flight (TOF) mass spectrometers, ion trap (IT) mass spectrometers, quadrupole mass spectrometers, and Fourier-transform ion cyclotron (FTICR or FT) mass spectrometers, and the recently introduced orbitrap. Recently, the FTICR mass spectrometer has emerged as the instrument of choice for biological studies due to its combination of flexibility, high mass accuracy, superior mass resolution, sensitivity, and wide dynamic range [5-9].

Another significant performance parameter of the FT mass spectrometer is its limit of detection. Limit of detection is defined by the minimum number of singly charged ions that can reliably be detected in the mass analyzer of the FT mass spectrometer, also known as the ion cyclotron resonance (ICR) cell [10-13]. By lowering the limit of detection, the dynamic range of the instrument is increased. This allows detection of low abundance compounds or fragment ion peaks, which often have biological significance. Another important implication of improving the detection limit of an FT mass spectrometer is improved mass accuracy via reduced space-charge induced frequency shifts [14]. The best that can be done is to be able to detect a single ion with unit charge, com-

Address reprint requests to Professor Peter B. O'Connor, Boston University, School of Medicine, Mass Spectrometry Resource, Department of Biochemistry, 670 Albany St., Boston University, Boston, MA2118, United States. E-mail: poconnor@bu.edu monly referred as unit charge detection limit [15]. Unit charge detection is routinely achieved in TOFMS; however, the lowest detection limit reported in literature for an FT mass spectrometer is 30 charges [16]. This manuscript presents an engineering approach to improve the limit of detection of an FT mass spectrometer.

In the ICR cell of an FTMS, ions are excited into a coherent cyclotron orbit by the application of a resonant or swept frequency rf voltage to the excitation electrodes. These coherent, rotating packets of ions induce image charges on the detection electrodes of the ICR cell [10]. The induced image current is amplified using a transimpedance amplifier. This detection amplifier is typically divided into two stages: (1), a first "preamplifier" stage, which is mounted sometimes in vacuum close to the detection plates of the ICR cell to minimize the capacitance, and (2), a second stage, which is usually placed outside the vacuum. The output of the second stage is sent to the analog-to-digital converter (ADC) that samples the analog signal and stores it in a digital format. Fast Fourier transform of the digital data yields the frequency domain spectrum of the ion signal. Finally, the frequency spectrum is converted into a mass spectrum using a calibration equation [17-19]. The detection plates, the amplifier, and the ADC constitute the detection circuitry of the FTMS. Enhancing the limit of detection involves careful analysis and optimization of these three components. Typically, it is the detection amplifier, which is engineered for optimal performance (low noise, high gain, low output impedance), given the existing ICR cell and the ADC specifications.

The equivalent electrical model for the detection scheme in the ICR experiments was presented by Comisarow [10]. In this approach, the resonantly excited 


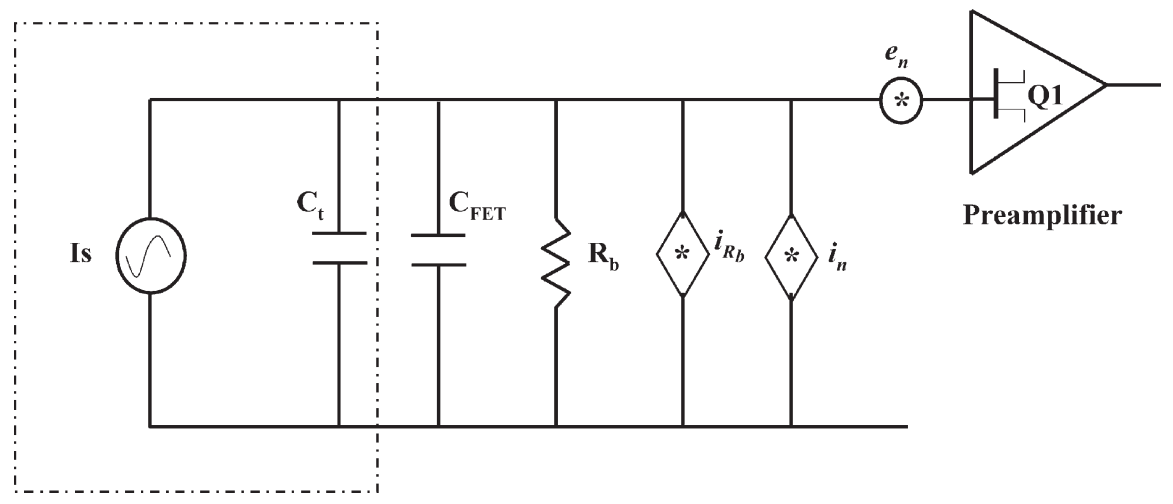

Figure 1. Equivalent electrical model for ICR detection $\left(I_{\mathrm{s}}=\right.$ induced image current, $C_{t}=C_{c}+C_{c w}$ $C_{c}=$ intrinsic cell capacitance, $C_{c w}$ is the connecting wire capacitance, $C_{F E T}=$ preamplifier input capacitance, $R_{b}=$ preamplifier input bias resistance, $i_{R b}=$ Johnson noise due to $R_{b}, i_{n}=$ preamplifier equivalent input noise current, $e_{n}=$ preamplifier equivalent input noise voltage).

coherent ion packet was modeled as a rotating electric monopole. Comisarow computed the expressions for the induced image current and voltage on the plates of a cubic ICR cell using his rotating monopole model. In the derivation it was assumed that the parallel detection plates were infinitely long. This assumption is naturally not true and error from this approximation becomes significant when the dimensions of the cell plates are comparable to the spacing between them. A comprehensive analytical solution for the induced differential charge in an ICR cell with arbitrary geometry was derived by Grosshans [20]. Using Green's function, Grosshans computed the magnitude of the Fourier coefficients of the signal induced by an ensemble of ions in a circular orbit. The equivalent electrical model makes it possible to develop an analytical solution for the design of the low noise preamplifier for ICR.

A pioneering step to enhance the limit of detection by improving the detection amplifier was taken by Anderson et al [16], which is further based on the work of Jefferts and Walls [21]. Anderson presented a design of a low noise amplifier for the FTMS using a JFET input differential stage. Various factors that determine the signal/noise ratio of the amplifier were analyzed.

The work presented here is a continuation of the investigations by Anderson et al. for the design of a low noise preamplifier for an FTMS. A detailed discussion of the design parameters involved for the ICR detection circuit is included. A low noise, wide bandwidth, differential transimpedance amplifier has been designed and implemented on a specially developed circuit board, and its performance has been evaluated on a custom FTICR mass spectrometer with a 7 Tesla superconducting magnet [8].

\section{Theory}

\section{ICR Signal}

Comisarow calculated the image current, $I_{s}$, induced on the plates of a cubic ICR cell (assuming infinitely long electrodes) due to the cyclotron motion of the ions, as given by eq 1 [10]. The theory was based on the work of Shockley, who calculated the induced current in electrodes in proximity of a moving charge [22].

$$
I_{s}(r . m . s)=\frac{N q^{2} r B}{\sqrt{2 m d}}
$$

where, $N$ is the number of ions with mass to charge ratio $m / q$, rotating in an orbit with cyclotron radius $r$ in a magnetic field of strength $B$, and $d$ is the distance between the plates of the ICR cell.

Now, the cyclotron frequency, $\omega_{c}$, is given by eq 2 .

$$
\omega_{c} \equiv \frac{q B}{m}
$$

Thus, using eq 2 in eq 1 ,

$$
I_{s}(r . m . s)=\left(\frac{N q r}{\sqrt{2} d}\right) \omega_{c}
$$

From the signal model presented in Figure 1, the voltage induced on the plates (i.e., at the input of the preamplifier), $V_{s}$ is given by,

$$
V_{s}=I_{s}\left(R_{b} \| X_{c}\right)
$$

where, $X_{c}$ is the reactance due to the total capacitance at the input of the preamplifier $C, R_{b}$ is the input bias resistor as defined in Figure 1.

$$
\left|X_{c}\right|=\frac{1}{\omega_{c}\left(C_{t}+C_{F E T}\right)} \equiv \frac{1}{\omega_{c} C}
$$

Hence, 


$$
V_{s}=\left(\frac{N q r \omega_{c}}{\sqrt{2} d}\right) \sqrt{\left(\frac{R_{b}^{2}}{1+\omega_{c}^{2} C^{2} R_{b}^{2}}\right)}
$$

If the bias resistor $R_{b}$ is selected such that: $R_{b} \gg X_{c}$ at minimum frequency of interest, then $\left(R_{b} \| X_{c}\right) \approx X_{c}$. Therefore,

$$
V_{s}=\left(\frac{N q r \omega_{c}}{\sqrt{2} d}\right)\left(\frac{1}{\omega_{c} C}\right)
$$

Thus, the r.m.s. induced signal voltage in the ICR cell is given by:

$$
V_{s}=\frac{N q r}{\sqrt{2} d C}
$$

As noted by Comisarow, the intriguing aspect of eq 8 is the absence of the frequency term, $\omega_{c}$. This independence of induced voltage on frequency ensures a flat response of the detection circuit, (over the working range in $\mathrm{m} / \mathrm{z}$ ) which simplifies ion quantification, provided the detection amplifier is designed with a flat gain in this bandwidth of interest. For a $7 \mathrm{~T}$ FT mass spectrometer, the relevant frequency range is generally from $10 \mathrm{kHz}(m / z \approx 10 \mathrm{k})$ to $1 \mathrm{MHz}(m / z \approx 100)$. Hence an $R_{b}$ greater than about $1 \mathrm{M} \Omega$ will ensure a flat response in this frequency range. This value of $R_{b}$ also determines the Johnson noise as discussed below.

From eq 8, the induced signal voltage is inversely proportional to the total capacitance, $\mathrm{C}$, at the input of the preamplifier which includes the detection plate capacitance $\left(C_{c}=10-20 \mathrm{pF}\right)$, stray capacitance of the wires connecting the preamplifier to cell plates $\left(C_{c w}=\right.$ 1-5 pF), and the intrinsic capacitance of the FETs used in the circuit of the preamplifier $\left(C_{F E T}=5-10 \mathrm{pF}\right)$. It is clearly best to minimize this capacitance, which can be done by reducing the size of the ICR cell, by mounting the preamplifier close to the detection plates, or by selecting a FET with minimum capacitance. However, if the ICR cell geometry is made smaller to reduce the capacitance, it leads to increased ion density in the ICR cell. This causes space charge effects, such as ion coalescence, which are detrimental to the mass accuracy and resolving power of the FTMS [17, 23, 24]. Moreover, the number of ions, $\mathrm{N}$, which can be efficiently trapped/excited/detected in the ICR cell, is reduced for a smaller cell. This causes reduction in induced ICR signal. Similarly, mounting the preamplifier in close proximity of the detection plates to minimize the lead wire capacitance is not always feasible. The ICR cell is placed in the homogenous high field region of the superconducting magnet. Most of the commercial low noise FETs do not operate well in such high magnetic fields, due to the Hall effect [25]. A possible solution is to design the preamplifier such that the FETs are mounted with their channel aligned to the magnetic field. However most of the commercial FETs, often with transverse package design, naturally prohibit such alignment. Finally, the intrinsic capacitance of the input FETs can be minimized by reducing the width of the channel, but with a respective trade-off, as discussed below.

\section{Input Equivalent Noise in an FTICR Detection Circuit}

Comisarow developed the electrical model of the ICR detection circuit shown in Figure 1. Using this model, the total mean square input equivalent noise voltage can be calculated.

$$
\bar{e}_{\mathrm{ni}}^{2}=\left(\bar{l}_{R_{b}}^{2}+\bar{\imath}_{n}^{2}\right)\left(\frac{X_{c} R_{b}}{X_{c}+R_{b}}\right)^{2}+\bar{e}_{n}^{2}
$$

where, $\bar{e}_{n i}$ is the total equivalent noise voltage at the input of the preamplifier. $\bar{l}_{R_{b}}$ is equivalent Johnson noise current spectral density due to $R_{b}$, equal to $\sqrt{4 k T \Delta f / R_{b}}$ [26], and $X_{c}$ is the source impedance due to total capacitance at the input of the preamplifier, equal to $1 / j \omega_{c} C$ (where $C=C_{t}+C_{F E T}$ ).

The value of capacitance to ground of a 3 in. diameter open cylindrical cell [27] is calculated [28] and measured at Boston University to be $\approx 10 \mathrm{pF}$ per plate. If the sum of input capacitance of the preamplifier and the connecting wires is $\approx 12 \mathrm{pF}$, the total capacitance at the input of the preamplifier becomes $\approx 22 \mathrm{pF}$.

The maximum value of impedance due to this capacitance will occur at the lowest cyclotron frequency of interest. For example for a 7 tesla FTMS at $10 \mathrm{kHz}$, this impedance will be:

$$
X_{c, \max }=530 \mathrm{k} \Omega
$$

Now, if the bias resistor in the ICR detection circuit is selected such that $R_{b} \gg X_{c, \max }$, then from eq 9,

$$
\begin{aligned}
\bar{e}_{n i}^{2} & \approx\left(\bar{\imath}_{R_{b}}^{2}+\bar{\imath}_{n}^{2}\right)\left|X_{c}\right|^{2}+\bar{e}_{n}^{2} \\
& \approx \frac{4 k T \Delta f}{R_{b}}\left(\frac{1}{\omega_{c} C}\right)^{2}+\left(\frac{\bar{\imath}_{n}^{2}}{\omega_{c}^{2} C^{2}}\right)+\bar{e}_{n}^{2}
\end{aligned}
$$

Thus, to minimize the noise voltage contribution of the bias resistor, the value of $R_{b}$ should be maximized.

The maximum value of $R_{b}$ which can be used is defined by the intrinsic input leakage current of the FET used in the preamplifier. The primary purpose of the $R_{b}$ in the preamplifier circuit is to set the d.c. bias at the gate of the input FET (and the detect plates) to ground potential. However, due to intrinsic leakage current from the gate of the FET, a charge starts to build up on the input capacitance of the preamplifier. This accumulation of charge floats the d.c. potential at the gate of this FET, eventually driving it to the power supply rails. Thus making the preamplifier nonoperational. Moreover, this leakage current also causes an ohmic voltage 
drop across $R_{b}$, which is directly connected to the detection plates and as a result will perturb ion motion. One volt of potential developed on the detection plates (10 pA of leakage current with a $100 \mathrm{G} \Omega$ bias resistor) can potentially cause a complete loss of detected ICR signal.

In this work $10 \mathrm{mV}$ was taken to be permissible on the detection plates and have been verified experimentally to be non detrimental for signal detection. As a result, the maximum value of $R_{b}$ that can be used with a FET with $10 \mathrm{pA}$ of leakage current is equal to $1 \mathrm{G} \Omega$. A general conclusion that can be made is to select a FET with minimum leakage current, thus permitting maximization of the $R_{b}$ and hence minimization of the input equivalent noise to the intrinsic noise of the input FET.

Increasing the value of $R_{b}$ to reduce its noise contribution only helps up to the point where the intrinsic noise of the FET, $e_{n}$, becomes significant. For example, the noise contribution of a $1 \mathrm{G} \Omega$ bias resistor at the input of the preamplifier is merely $0.6 \mathrm{nV} / \mathrm{rtHz}$ at 100 $\mathrm{kHz}(\mathrm{T}=290$ Kelvin, and $\mathrm{C}=10 \mathrm{pF})$. This is already below the input noise voltage of most of the commercial low noise JFETs used for the ICR preamplifier designs. Thus, increasing the bias resistor further does not enhance the net ICR signal/noise ratio.

\section{Input Device Selection}

The above discussion shows that the selection of the input FET is the most significant design choice for the optimization of the ICR detection preamplifier. The ICR signal is modeled as a current source in parallel with a capacitance [10]. In such a high impedance source, the leakage current of the input transistor can potentially have a dominant influence on the input-referred electronic noise. Thus, it is desirable to use junction field effect transistors (JFETs) at the input stage, which have lower leakage currents than bipolar junction transistors (BJTs) [21]. JFETs have leakage currents on the order of $1 \mathrm{pA}$, allowing a high input bias resistor, which helps minimize noise as discussed above. Moreover, the low $1 / \mathrm{f}$ noise cut-off frequency in JFETs, typically below 1 $\mathrm{kHz}$, has significant implication in low noise ICR detection where spectral frequencies of interest are usually above $10 \mathrm{kHz}$.

Noise sources in a JFET can be attributed to the following sources [29]:

- $1 / \mathrm{f}$ noise caused by generation recombination of charge carriers in the depletion region of the FET. In modern JFETs the 1 / f noise roll-off is typically below a $\mathrm{kHz}$, hence this component of noise is neglected at higher frequencies.

- Shot noise due to the leakage current of the gate of the JFET. This can be calculated by the Schottky formula $I_{\text {shot }}{ }^{2} / \Delta f=2 q I_{G S S}$, where $q$ is the charge of an electron, $I_{G S S}$ is the gate leakage current [30].
- Channel noise due to thermal fluctuations in the drain to source channel. This noise can be calculated by determining the equivalent value of the channel resistance as shown below.

- Channel thermal noise, capacitively-coupled to gate (input) by $C_{g d}$. This noise is represented as a shunt resistance $\left(R_{n} \approx 1 /\left(\omega^{2} r_{d s}^{2} g_{m} C_{g d} C_{d s}\right)\right)$ in parallel with the $C_{g s}$ at the input of the FET. The contribution of the thermal noise current due to $R_{n}$ is typically less than $10 \mathrm{fA} / \mathrm{rtHz}$ at frequencies below $1 \mathrm{MHz}$, becoming significant only at higher frequencies and is neglected for most practical applications [29, 32].

Thus, in the ICR frequency range of operation, the primary contribution to the noise of the JFET comes from the thermal noise in the channel. Hence, to obtain optimum noise performance it is proper to consider channel thermal noise of the device, as discussed below.

The ICR signal arises by integrating the image current over the total input capacitance consisting of cell plates, connecting wires, and the JFETs input capacitance. Hence, reducing this capacitance increases the ICR signal, which is evident from eq 8 . Again, as the geometry of the ICR cell plates is fixed, it is of interest to minimize the connecting wire capacitance and the JFET input capacitance for maximizing the ICR signal. The wire capacitance is minimized by placing the preamplifier in vacuum, near the ICR cell. However, the electronic noise of a JFET, which primarily consists of the Johnson noise in the intrinsic channel resistance, increases as the JFET intrinsic capacitance decreases or, in other words, as the device is made smaller. As a result, the scaling down of the device to reduce the capacitance makes it noisier. Selection of a JFET with an input capacitance for optimal ICR signal/noise ratio can be done on the basis of the following analysis.

The JFET equivalent channel noise resistance between drain and source terminal in the saturation region is $3 /\left(2 \mathrm{~g}_{\mathrm{m}}\right)$, where $g_{m}$ is the transconductance of the JFET [33]. As a result, the equivalent input noise voltage in a JFET (referred to the gate) can be expressed as:

$$
\bar{v}_{n}^{2}=4 k T\left(2 /\left(3 g_{m}\right)\right) \Delta f
$$

Hence, using eq 8, the signal/noise ratio (signal power/ noise power) of the ICR signal at the input of the preamplifier can be calculated by (ignoring the JFET noise current contribution as discussed above),

$$
\text { signal/noise }=\frac{V_{s}^{2}}{\bar{v}_{n}^{2}}=\left(\frac{N q r}{\sqrt{2} d\left(C_{t}+C_{F E T}\right)}\right)^{2}\left(\frac{3 g_{m}}{8 k T \Delta f}\right)
$$


Eq 12 can be rearranged as:

$$
\begin{aligned}
& \text { signal/noise } \\
& =\left(\frac{3 N^{2} q^{2} r^{2}}{16 d^{2} k T \Delta f}\right) \times\left(\frac{g_{m}}{C_{F E T}}\right) \times\left(\frac{1}{C_{F E T}\left(1+C_{t} / C_{F E T}\right)^{2}}\right)
\end{aligned}
$$

The transconductance, $g_{m}$, and the input capacitance of the JFET, $C_{F E T}$, both scale linearly with the width of the FET channel for a given device fabrication process [33]. This ratio of $g_{m}$ to $C_{F E T}$ is generally used as a figure of merit to compare various device processes used for the fabrication of a JFET. Therefore, the set of terms in the first two parenthesis are constant for a given ICR signal and a given device process. To find optimal device geometry, taking the derivative of the last term with respect to $C_{F E T}$ and equating to zero, gives:

$$
C_{t}=C_{F E T}
$$

Therefore, in ICR signal detection, the optimal signal/ noise ratio is obtained when the total capacitance due to the cell plates and connecting wires is minimized and matches the total input intrinsic capacitance of the JFET. Hence, in the preamplifier design, a JFET with a capacitance slightly smaller $(\approx 2 \mathrm{pF}$ to account for the connecting wire capacitance) than that of the capacitance of the cell plates is selected and with a device process for maximum $g_{m} / C_{F E T}$ ratio.

\section{Methods}

\section{Circuit Design}

The ICR detection amplifier is divided into 2 stages: an in vacuum preamplifier and a second stage amplifier, which is at atmospheric pressure. The preamplifier is used primarily to convert the high impedance of the ICR signal to a low impedance, with a corresponding current gain and very low noise. Further, the voltage amplification, which requires more power, is done outside the vacuum. This is done because the extremely low pressures in the FTMS (order of $10^{-10} \mathrm{mbar}$ ) hinders efficient cooling of the devices, which ultimately can cause power derating or device failure. Thus, most of the signal amplification is done in the second stage. However, the equivalent input noise of the ICR detection circuit is primarily defined by the in-vacuum preamplifier.

The ICR signal is inherently a differential signal and, thus, the current amplifier is a differential design. The differential configuration enables the extraction and amplification of the difference between two small input signals and cancels the common mode signal that both inputs share (which, for example, can come from $60 \mathrm{~Hz}$ interference, etc). A close symmetry (matching) of the components in the two legs of the differential amplifier is required for a high common mode rejection ratio, and because commercial JFETs show wide variation in characteristics, several of these were tested to find a pair with closely matched DC characteristic curves.
The circuit diagram of the BUSM (Boston University School of Medicine) in-vacuum preamplifier is shown in Figure 2. The cascode configuration was used to reduce the Miller effect, hence enhancing the bandwidth of the preamplifier [31]. In a simple differential amplifier without a cascode, the capacitance at the gate of the input transistor becomes $C_{g s}+(1+A) C_{g d}$, where $\mathrm{A}$ is the voltage gain from gate to drain. This apparent amplification of the $C_{g d}$ is due to the negative feedback from drain to gate of the input FET. This effect was first defined by John M. Miller, hence commonly referred as the Miller effect and is responsible in limiting the bandwidth of such an amplifier configuration [31]. In a cascode configuration the voltage gain from gate to drain is almost 1 at the input FET. Here the voltage at the source is in phase with the drain, thus eliminating negative feedback and minimizing the Miller effect. Hence, the apparent increase in $C_{g d}$ is avoided. The amplification is achieved by the cascode device, which is in a common-gate configuration.

The gate of the cascode device is tied to the source of the input device, to minimize the input gate leakage current. The source to drain voltage of Q1 and Q2 now becomes the gate to source voltage of the Q3 and Q4. This requires for the drain to source voltage of the input devices (Q1, Q2) to be much less in magnitude than the pinch off voltage of the cascode devices (Q3, Q4). This configuration limits the drain to source voltage of Q1, Q2 to small values, which keeps the leakage current negligible in the JFETs, independent of the drift in the supply voltage.

Emitter followers, Q5 and Q6, are used for impedance transformation of the ICR signal (from high to low). Low impedance on the long lines running from preamplifier to the electrical feed-through makes the amplifier less susceptible to interference pick up and noise coupling. Once the impedance level of the signal is low, a generic low noise operational amplifier (opamp) can be used for further amplification. A low noise instrumentation amplifier configuration is used for this purpose, using standard opamps as shown in Figure 3. Finally, the output of the amplifier is fed to an ADC for digitization.

\section{Results}

\section{On Bench Circuit Testing}

JFETs from NXP Inc. (Eindhoven, The Netherlands), BF862, were used as input transistors. Matched JFETs U431 from Vishay Intertechnology, Inc. (Malvern, PA) were used as the cascode devices. BF862 has an input capacitance of $10 \mathrm{pF}$ and input noise voltage of 0.8 $\mathrm{nV} / \mathrm{rtHz}$, and represents the optimal trade off between noise and input capacitance. Low leakage JFETs and the above configuration allowed the use of high value input bias resistors, $1 \mathrm{G} \Omega$, to elevate the signal/noise ratio. BF862s were biased at VDS $=1.5$ volts and IDS $=8 \mathrm{~mA}$ giving a $g_{m}$ of approximately $35 \mathrm{mS}$. The $750 \Omega R_{d}$ provided a voltage gain of approximately 25 in the first stage. The emitter follower reduced the signal impedance 


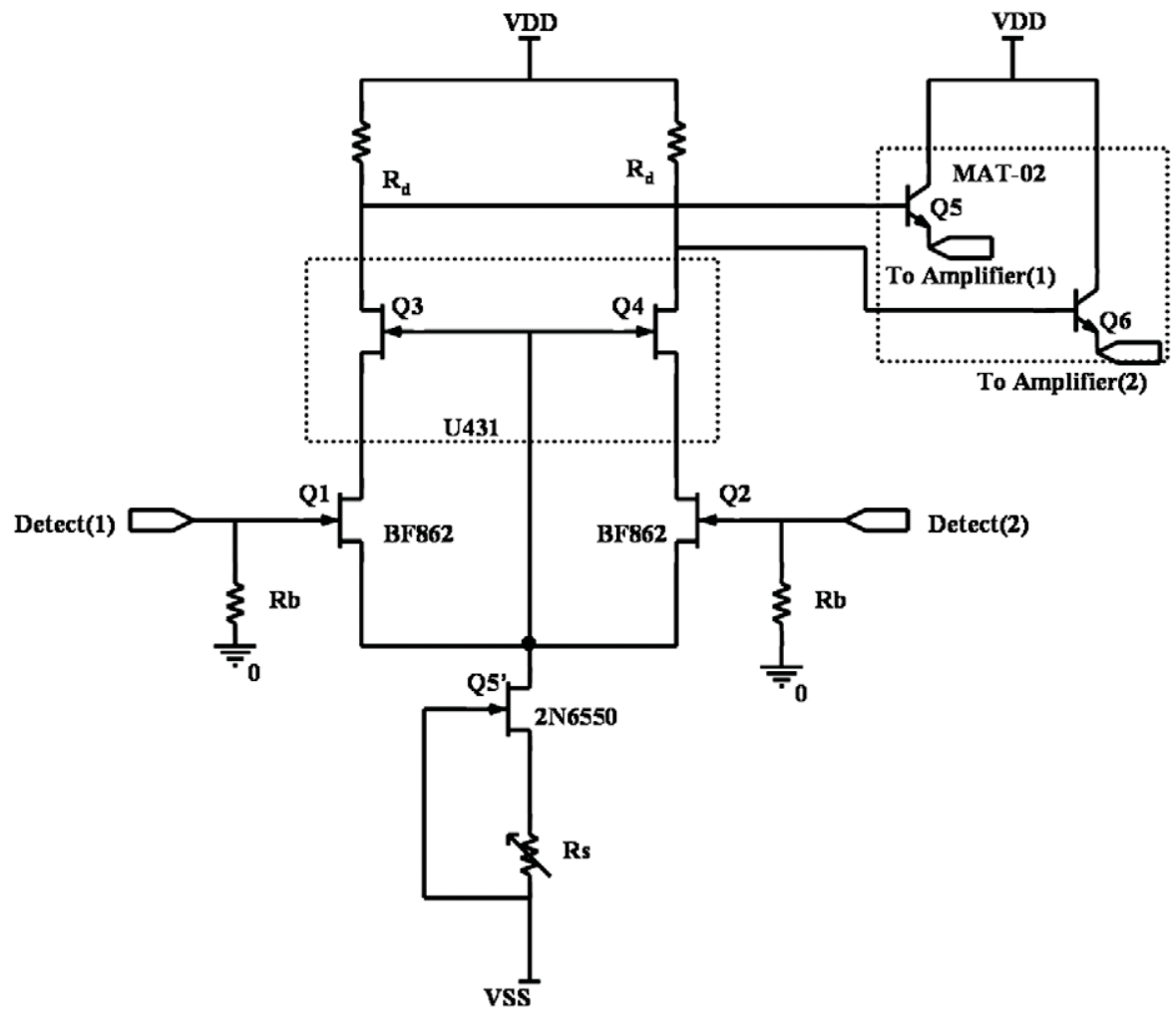

Figure 2. Schematic of the BUSM low noise wideband transimpedance preamplifier.

to approximately $50 \mathrm{ohms}$, primarily due to the balancing potentiometer at the emitter of Q5 and Q6. The constant current sources, Q7-Q9, kept the base-emitter voltage of Q5 and Q6 equal. This ensures that the current gain (CMRR/differential gain) is identical for each signal line.

The voltage gain versus frequency characteristic plot of the BUSM amplifier is shown in Figure 4 . The $-3 \mathrm{~dB}$ high frequency roll-off of the transimpedance amplifier is at $2.81 \mathrm{MHz}$, which corresponds to approximately 30
Da on a 7 tesla FTMS. The midband voltage gain of the amplifier is $\approx 3500$.

The electrical noise performance of the BUSM transimpedance amplifier was compared with that of a com mercial amplifier (Figure 5). The commercial amplifier consists of a unity gain source follower, using OPA637, as a preamplifier followed by a high gain instrumentation amplifier. The midband gain of the commercial amplifier is 1000. The BUSM transimpedance amplifier was pow-

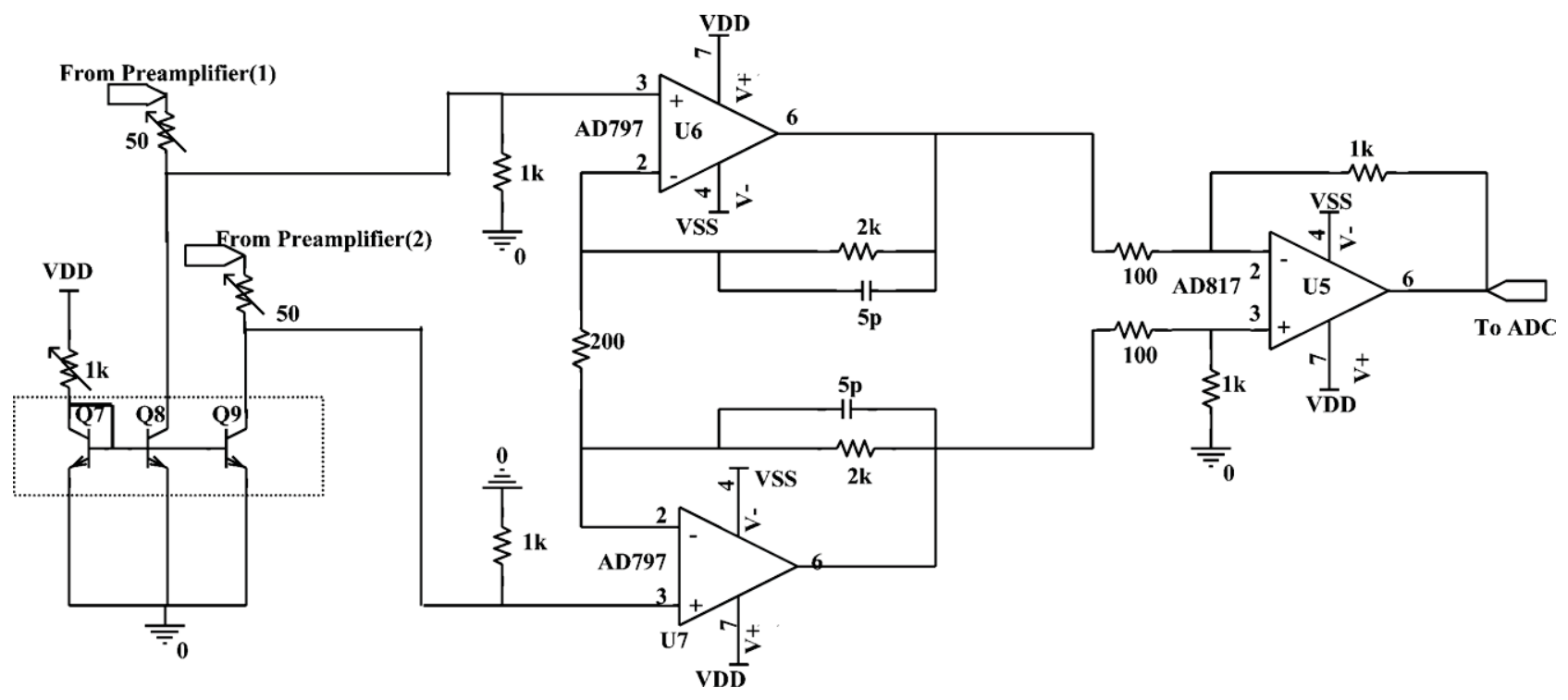

Figure 3. Schematic of the BUSM instrumentation amplifier. 


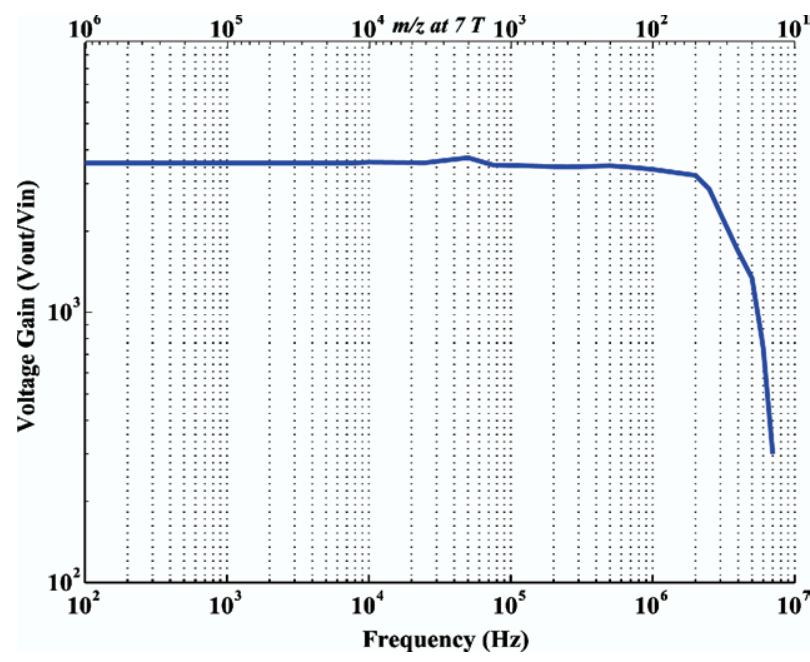

Figure 4. Gain bandwidth plot of the low noise wideband BUSM transimpedance amplifier.

ered either by switching power supplies (Agilent Technologies, Santa Clara, CA) with unshielded cables in the room or by $9 \mathrm{~V}$ batteries. The commercial amplifier was powered by linear power supplies (Varian Inc., Lakeforest, CA).

The equivalent input rms noise voltage was measured by shorting the inputs of the preamplifiers to ground. A Tektronix spectrum analyzer (Tektronix 2712, Richardson, TX) was used to obtain the short circuit output noise spectrum from $10 \mathrm{kHz}$ to $1 \mathrm{MHz}$. To compare the two amplifiers, the output noise spectrum was divided by the corresponding voltage gain spectrumto obtain the input equivalent rms noise voltage density, as shown in Figure 5. The BUSM transimpedance amplifier has shown approximately 40 times lower rms noise voltage using the batteries (at $100 \mathrm{kHz}$ ), compared with the commercial amplifier. Also, the $1 / \mathrm{f}$ noise corner frequency is more than 10 times lower in the case of the BUSM transimpedance amplifier.

\section{Instrument Testing}

A custom MALDI-FTMS was used to evaluate the low noise ICR amplifier [8]. The instrument has an open cylindrical ICR cell geometry with 3 in. o.d. The preamplifiers were mounted close to the detection plates minimizing the connecting wire parasitic capacitance. Magnet wire from McMaster-Carr (Princeton, NJ), which is enamel coated copper, is used for all in-vacuum electrical connections. The outputs from the in-vacuum preamplifier are connected to the instrumentation amplifier via a con-flat BNC electrical feed-through (MDC Vacuum Products, Hayward, CA). It is always recommended to remove any noise component outside the desired bandwidth using a filter. Thus, a low pass, 11th-order Butterworth filter from TTE Inc. (Los Angeles, CA) is used before the digitizer to remove any unwanted noise above $1 \mathrm{MHz}$. Several sets of mass spectra were obtained to compare the signal/noise ratio of the current amplifier with the commercial one.

$\mathrm{C}_{60}$ was used as a standard for the studies on a custom MALDI-FTMS [8]. A $1 \mu \mathrm{M}$ solution of $\mathrm{C}_{60}$ in toluene was spotted onto a stainless steel plate and allowed to dry. Spectra were generated using a single shot of a $355 \mathrm{~nm}$

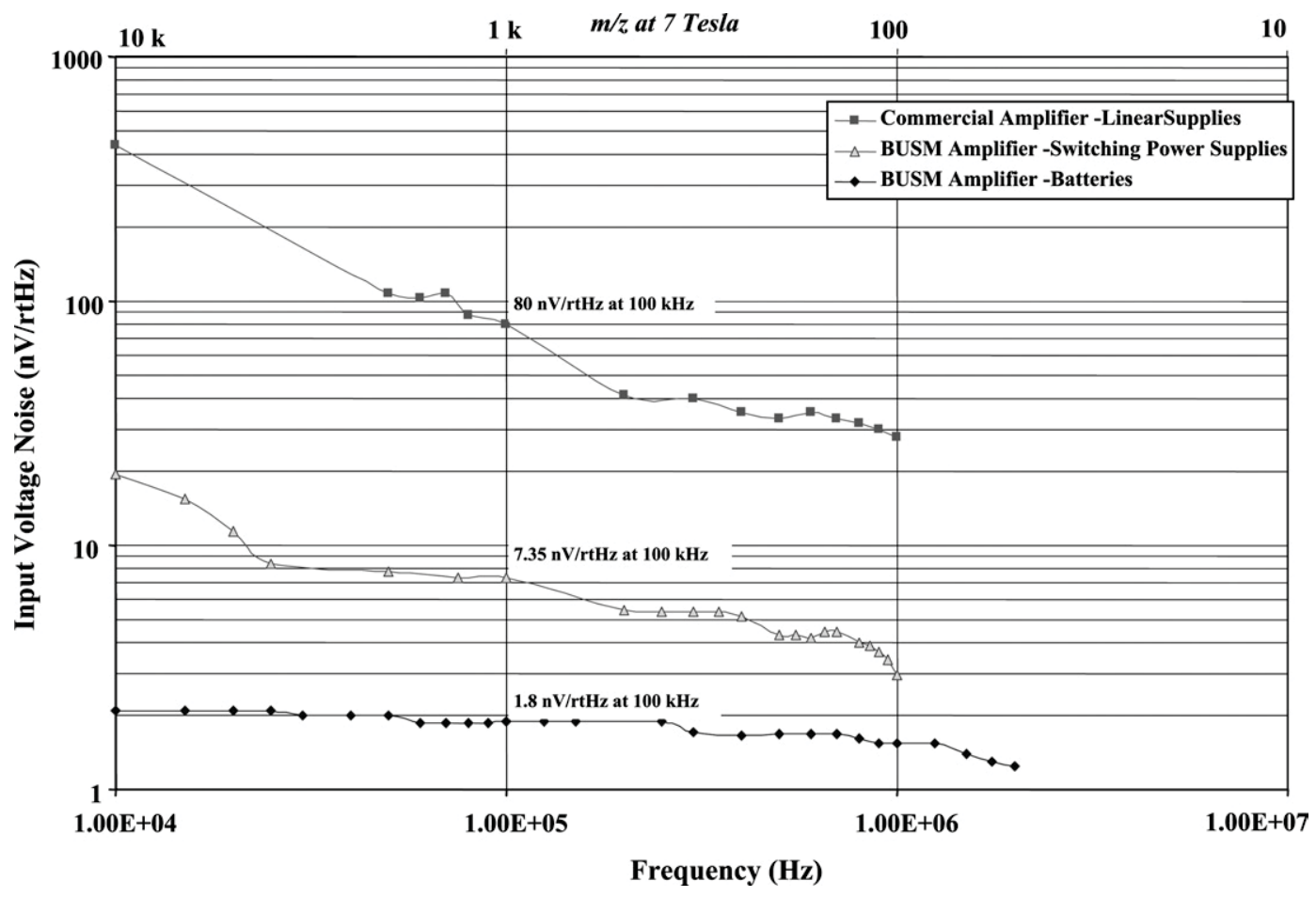

Figure 5. Equivalent input r.m.s. noise voltage density of the two amplifier designs. 


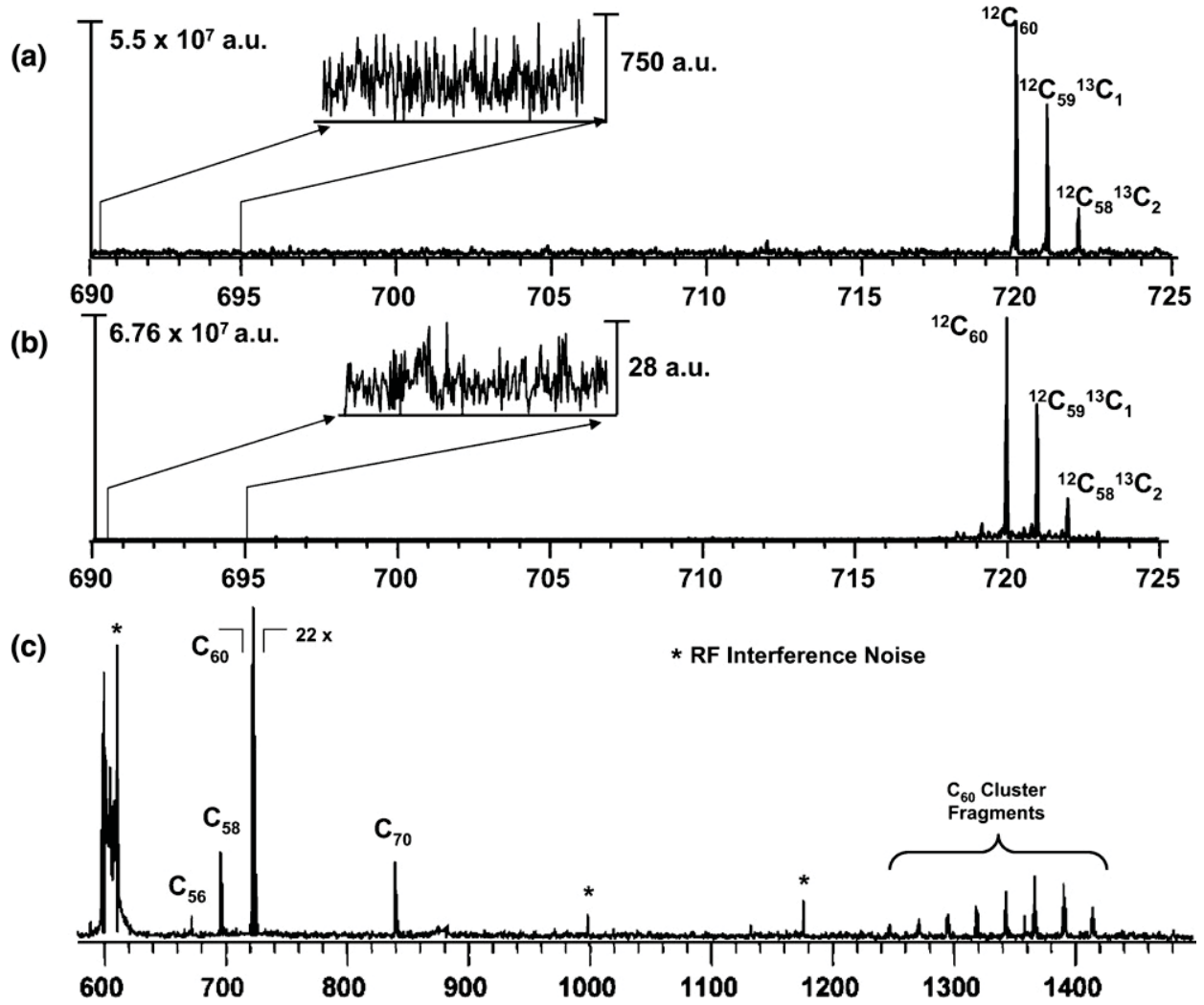

Figure 6. MS of $\mathrm{C}_{60}$ obtained using two amplifiers with identical instrument settings on a MALDIFTMS. Input referred baseline noise is shown in the inset. MS acquired with BUSM transimpedance amplifier powered by switching power supplies in (b) has $\approx 25$-fold reduction in noise compared with that of commercial amplifier in (a); (c) shows the complete broadband $\mathrm{m} / \mathrm{z}$ range of the MS in (b) using the BUSM amplifier.

Nd:YAG laser from Continuum Inc. (Santa Clara, CA.) at $50 \mu \mathrm{J} /$ pulse. The ions were transferred to the ICR cell using a pair of hexapoles driven by high voltage RF oscillators [34, 35] and were trapped using gated trapping. After $50 \mathrm{~ms}$ of thermal stabilization, the ions were resonantly excited into coherent cyclotron orbits by the application of a broadband RF sweep. The RF sweep voltage of $200 V_{p-p}$ was applied for $8 \mathrm{~ms}$ and swept from $150 \mathrm{Da}$ to 3000 Da. The excitation was followed by image current detection using the amplifier under test; $512 \mathrm{~K}$ samples were taken from the amplified ICR signal at a rate of 2 $\mathrm{MHz}$ (total transient length of $0.262 \mathrm{~s}$ ). The digitized data was zero filled to $1024 \mathrm{~K}$ points and fast Fourier transformed without apodization. The resulting magnitude mode frequency spectra were converted to a mass spectra and are shown in Figure 6. The detected signal, using the BUSM low noise differential amplifier, Figure $6 \mathrm{~b}$, demonstrated approximately a 25-fold reduction in noise compared to the commercial one, Figure 6a. In this comparison, the commercial in-vacuum amplifier was powered with linear power supplies, but the BUSM transimpedance preamplifier was powered with unshielded switching power supplies. Subsequent tests showed an additional $\approx 4$-fold reduction in noise when the power supplies were swapped to a shielded battery powered supply (Figure 5). The plots in Figure 5 represent the short circuit input noise, while this $C_{60}$ comparison is for an open, capacitive input impedance, so the plots are not directly comparable. Although Figure 6 shows an apparent $\approx 30$ fold improvement in signal/noise ratio, due to shot-toshot instability in laser desorption/ionization, this may be a less useful comparison. It should be noticed that the family of peaks which appear besides the $C_{60}$ isotopes in Figure $6 \mathrm{~b}$ are due to several reasons; namely, the characteristic Lorentzian line shape of the isotopic peaks, Gibbs oscillations, and the mixing of axial/magnetron motion frequencies (sidebands) to the cyclotron frequency. Furthermore, RF interference noise can cause (and did in this case) a transient to "clip," which generates further mixing artifacts. The symmetry of these peaks on either side of the isotopic peaks indicate that these are not random thermal noise peaks characterizing the base line noise of the BUSM amplifier. Figure $6 c$ shows the complete broadband $\mathrm{m} / \mathrm{z}$ range of the mass spectrometer in Figure $6 \mathrm{~b}$ obtained with the BUSM amplifier.

\section{Conclusion}

The Fourier transform mass spectrometry has shown unmatched performance in biological and chemical studies due to its high mass accuracy, resolving power, and dynamic range. These FTMS capabilities can be signifi- 
cantly improved by enhancing the signal/noise ratio of its detection circuit which includes the ICR cell, detection preamplifier, amplifier, and the digitizer. For an existing ICR cell and digitizer, optimization of the detection preamplifier becomes a non-trivial design problem. In this article we have discussed an analytical approach involved in the development of such a low noise FTMS detection amplifier.

Following the electrical model of ICR detection by Comisarow, expressions for the signal/noise ratio in ICR detection are presented and show that cell plate and the connecting wire capacitance should be minimized. Furthermore, it is shown that the FETs for the preamplifier should be selected with minimum input leakage current. This allows higher values of the input bias resistor, thus enhancing the signal/noise ratio of the ICR detection circuit. Moreover, the FET input capacitance should be matched to the sum of capacitance (to ground) from the detection plates and the connecting wires for optimal signal/noise ratio. It is also recommended to mount the preamplifier in vacuum in close proximity to the detection plates to reduce the effect of parasitic capacitance from the connecting wires.

An improved transimpedance detection amplifier has been designed for an FT mass spectrometer. The electrical parameters of the current design were characterized on the bench and showed a mid-band voltage gain of 3500 with a $3 \mathrm{~dB}$ bandwidth of $2.81 \mathrm{MHz}$. The input rms noise performance of the transimpedance amplifier was compared to that of a current commercial amplifier using a spectrum analyzer. The transimpedance amplifier showed a 40-fold lower input rms noise voltage using battery powered supply voltages.

Evaluation and comparison of the amplifiers on a MALDI-FT mass spectrometer was conducted using $\mathrm{C}_{60}$. Even using switching power supplies, a mass spectrum of $\mathrm{C}_{60}$ obtained using the new transimpedance amplifier exhibited $\mathrm{a} \approx 25$ times lower noise compared to the one from the existing commercial amplifier and 30-fold higher signal/noise ratio.

Currently, experiments to determine the limit of detection of the FT mass spectrometer, using the low noise transimpedance amplifier, are being conducted. An ESIFTMS and an ion number estimating algorithm developed in-house will be used for this purpose [9, 13].

\section{Acknowledgments}

The authors acknowledge Dr. Alan Rockwood and Dr. Saikat Ray for useful discussions. The help from Konstantine Aizikov and Xiaojuan Li in instrumental set-up is greatly appreciated. This work was sponsored by NIH/NCRR P41RR10888, by NIH/NHLBI N01HV28178, and by MDS-Sciex.

\section{References}

1. Aebersold, R.; Goodlett, D. R. Mass Spectrometry in Proteomics [Review]. Chem. Rev. 2001, 101, 269-295.

2. Gygi, S. P.; Han, D. K.; Gingras, A. C.; Sonenberg, N.; Aebersold, R. Protein Analysis by Mass Spectrometry and Sequence Database Searching: Tools for Cancer Research in the Post-Genomic Era. Electrophoresis 1999, 20, 310-319.
3. Mann, M.; Hojrup, P.; Roepstorff, P. Use of Mass Spectrometric Molecular Weight Information to Identify Proteins in Sequence Databases. Biol. Mass Spectrom. 1993, 22, 338-345.

4. Cronshaw, J. A.; Krutchinsky, A. N.; Zhang, W. Z.; Chait, B. T.; Matunis, M. J.; Yates, J. R. Proteomic Analysis of the Mammalian Nuclear Pore Complex. J. Cell Bio. 2002, 158, 915-927.

5. Comisarow, M. B.; Marshall, A. G. Fourier Transform Ion Cyclotron Resonance Spectroscopy. Chem. Phys. Lett. 1974, 25, 282-283.

6. Comisarow, M. B.; Marshall, A. G. Frequency-Sweep Fourier Transform Ion Cyclotron Resonance Spectroscopy. Chem. Phys. Lett. 1974, 26, 489-490.

7. Amster, I. J. Fourier Transform Mass Spectrometry. J. Mass Spectrom. 1996, 31, 1325-1337.

8. O'Connor, P. B.; Budnik, B. A.; Ivleva, V. B.; Kaur, P.; Moyer, S. C.; Pittman, J. L.; Costello, C. E. A High Pressure Matrix-Assisted Laser Desorption Ion Source for Fourier Transform Mass Spectrometry Designed to Accommodate Large Targets with Diverse Surfaces. J. Am. Soc. Mass Spectrom. 2004, $15,128-132$

9. O'Connor, P. B.; Pittman, J. L.; Thomson, B. A.; Budnik, B. A.; Cournoyer J. C.; Jebanathirajah, J.; Lin, C.; Moyer, S.; Zhao, C. A New Hybrid Electrospray Fourier Transform Mass Spectrometer: Design and Performance Characteristics. Rapid Commun. Mass Spectrom. 2006, 20, 259-266.

10. Comisarow, M. B. Signal Modeling for Ion Cyclotron Resonance. J. Chem. Phys. 1978, 69, 4097-4104.

11. Comisarow, M. B.; Marshall, A. G. Theory of Fourier Transform Ion Cyclotron Resonance Mass Spectroscopy. J. Chem. Phys. 1976, 64, 110-119.

12. Limbach, P. A.; Grosshans, P. B.; Marshall, A. G. Experimental Determination of the Number of Trapped Ions, Detection Limit, and Dynamic Range in FTICR-MS. Anal. Chem. 1993, 65, 135-140.

13. Kaur, P.; O'Connor, P. B. Use of Statistical Methods for Estimation of Total Number of Charges in a Mass Spectrometry Experiment. Anal. Chem. 2004, $76,2756-2762$.

14. Easterling, M. L.; Mize, T. H.; Amster, I. J. Routine Part-Per-Million Mass Accuracy for High-Mass Ions: Space-Charge Effects in MALDI FT-ICR. Anal. Chem. 1999, 71, 624-632.

15. Difilippo, F.; Natarajan, V.; Bradley, M.; Palmer, F.; Pritchard, D. Accurate Atomic Mass Measurements from Penning Trap Mass Comparisons of Individual Ions. Phys. Scripta 1995, 63, 144-154.

16. Anderson, G. A.; Bruce, J. E.; Hofstadler, S. A.; Rockwood, A.; Smith, R.D. Low noise ICR detection electronics. Proceedings of the 43rd Annual Conference of the American Society for Mass Spectrometry, Atlanta, GA, May 1995.

17. Francl, T.; Sherman, M. G.; Hunter, R. L.; Locke, M. J.; Bowers, W. D.; McIver, R. T. Experimental Determination of the Effects of Space Charge on Ion Cyclotron Resonance Frequencies. Int. J. Mass Spectrom. Ion Processes 1983, 54, 189-199.

18. Ledford, E. B.; J., Rempel, D. L.; Gross, M. L. Space Charge Effects in Fourier Transform Mass Spectrometry. Mass Calibration Anal. Chem. 1984, $56,2744-2748$.

19. Zhang, L. K.; Rempel, D.; Pramanik, B. N.; Gross, M. L. Accurate Mass Measurements by Fourier Transform Mass Spectrometry. Mass Spectrom. Rev. 2005, 24, 286-309.

20. Grosshans, P. B.; Shields, P. J.; Marshall, A. G. Comprehensive Theory of the Fourier Transform Ion Cyclotron Resonance Signal for All Ion Trap Geometries. J. Chem. Phys. 1991, 94, 5341-5352.

21. Jefferts, S. R.; Walls, F. L. ALow-Noise Cascode Amplifier. J. Res. Nat. Bur. Stand. 1987, 92, 383-386.

22. Shockley, W. Currents to Conductors Induced by a Moving Point Charge. J. Appl. Phys. 1938, 9, 635-636.

23. Amster, I. J.; Kristina, T. P. Space Charge Effects on Mass Accuracy for Multiply Charged Ions in ESI-FTICR. Int. J. Mass Spectrom. 2003, 222, 351-361.

24. Jeffries, J. B.; Barlow, S. E.; Dunn, G. H. Theory of Space Charge Shift of ICR Frequences. Int. J. Mass Spectrom. Ion Processes, 1983, 54, 169-187.

25. Edwin Hall. On a New Action of the Magnet on Electric Currents. Am. J. Math. 1879, 2, 287-292.

26. Johnson, J. B. Thermal Agitation of Electricity in Conductors. Phy. Rev. 1928, 32, 97-109.

27. Beu, S. C.; Laude, D. A., J. Open Trapped Ion Cell Geometries for Fourier Transform Ion Cyclotron Resonance Mass Spectrometry. Int. J. Mass Spectrom. Ion Processes 1992, 112, 215-230.

28. Nikolaev, E. N.; Gorshkov, M. V. ICR Detection in an Infinite Cylindrical Trap. Int. J. Mass Spectrom. Ion Processes 1985, 64, 115-125.

29. Van Der Ziel, A. Noise sources, characterization, measurement; Prentice-Hall: Englewood, NJ 1970.

30. Schottky, W. Über Spontane Stromschwankungen In Verschiedenen Elektrizitätsleitern. Ann. Phys. 1918, 57, 541-568.

31. Miller, J. M. Dependence of the Input Impedance of a Three-Electrode Vacuum Tube upon the Load in the Plate Circuit. Scientific Papers of the Bureau of Standards 1920, 15, 367-385.

32. Levinzon, F. A. Noise of the JFET Amplifier. IEEE T Circuits Syst. 2000, 47, 981-985.

33. Gray, P.; Hurst, P.; Lewis, S.; Meyer, R. Analysis and Design of Analog Integrated Circuits; Wiley: Hoboken, NJ 2001; pp. 761-768.

34. Mathur, R.; O'Connor, P. B. Design and Implementation of a High Power rf Oscillator on a Printed Circuit Board for Multipole Ion Guides. Rev. Sci. Instrum. 2006, 77, 114101.

35. O'Connor, P. B.; Costello, C. E.; Earle William, E. A High Voltage rf Oscillator for Driving Multipole Ion Guides J. Am. Soc. Mass Spectom. 2002, 13, 1370-1375. 